\title{
Paradox of age-specific incidence rates of second primary cancer in individuals with esophageal cancer
}

\author{
Tomonori Yano 1
}

Received: 5 March 2020/ Accepted: 2 April 2020/Published online: 13 April 2020

(C) Japanese Society of Gastroenterology 2020

Keywords Esophageal cancer - Second primary cancer . Endoscopic monitoring - Head and neck cancer - Agespecific incidence rate

Aging must be the leading cause of cancer in clinical. In recent, translational research using samples of esophageal epithelium suggests that carcinogenesis of esophageal squamous cell carcinoma (ESCC) is related to age-related expansion of NOTCH1 mutated clones associated with exposure to large amounts of tobacco and alcohol consumption [1].

A recently published study by Maekawa and colleagues [2] revealed a high incidence of second primary cancer (SPC) in individuals who had undergone endoscopic resection (ER) for esophageal cancer. They conducted a retrospective analysis of a large cases with long-term follow-up to assess the incidence rate of SPC after ER. They found that the incidence rate of second primary head and neck squamous cell carcinoma (HNSCC) was significantly higher in patients under 60 years, however the age-specific incidence rate of other SPC including gastric cancer (GC) was the opposite. They recommended endoscopic monitoring of the laryngo-pharyngeal region of younger patients to ensure early cancer detection.

HNSCC is known the most common SPC in individuals with ESCC. Prognosis among individuals who developed HNSCC after esophagectomy was poor because of

Tomonori Yano

toyano@east.ncc.go.jp

1 Department of Gastroenterology and Endoscopy, National Cancer Center Hospital East, 6-5-1, Kashiwanoha, Kashiwa 277-8577, Japan difficulty in early detection [3]. However, as with other cancers of the gastrointestinal tract, HNSCCs in patients with a history of ESCC can be diagnosed early using narrow band imaging (NBI) endoscopy [4]. The efficacy of endoscopic monitoring using NBI of the laryngopharyngeal region for early detection of SPC has been demonstrated in a randomized controlled trial (RCT) [5]. Based on the favorable results of this RCT, we introduced endoscopic monitoring using NBI to our practice, and the number of diagnoses of superficial HNSCC has increased in recent as described in Maekawa's article. Another retrospective study revealed that endoscopic monitoring of laryngopharyngeal region may decrease mortality and complications related to metachronous advanced HNSCC in patients with ESCC [6]. Therefore, incidence rates of HNSCC in individuals with ESCC should be evaluated in the cohort after the era of the introduction of endoscopic monitoring using NBI.

Katada and colleagues conducted the important cohort study with intensive regular endoscopic monitoring to determine the incidence rate of metachronous ESCC and HNSCC after ER among patients with ESCC, and to identify risk factors for SPC [7]. They found the high cumulative incidence rates of ESCC and HNSCC, particularly in patients with multiple dysplastic esophageal epithelial lesions due to heavy alcohol and tobacco consumption. Furthermore, long-term follow-up for approximately 50 months revealed that abstaining from alcohol might reduce risk of aero-digestive SPC. Another longterm follow-up study of the risk of SPC after ER for ESCC according to genetic polymorphisms of alcoholdehydrogense-1B (ADH1B) and aldehyde dehydrogenase-2 (ALDH2) [8]. They found that inactive heterozygous ALDH2 and alcohol consumption were independent risk factors for second primary ESCC. However, genetic 
polymorphisms of slow-metabolizing ADH1B, not inactive heterozygous ALDH2 were the significant risk factors for HNSCC. Although age positvely correlated with second primary SCC only in esophagus, patients who were detected second primary SCC were significantly younger than patients without second primary SCC. These findings including Maekawa's report [2] demonstrated the importance of careful follow-up of young ESCC patients with slow-metabolizing ADH1B, who do not stop alcohol consumption after ER, by means of endoscopic monitoring of the laryngopharyngeal region combined with holistic support from a team consisting of an otolaryngologist, a psychiatrist, and social workers.

In contrast to HNSCC, the incidence of GC increases with age, and the incidence of a second primary GC was significantly higher in patients aged 60 years or older, than in younger patients in Dr. Maekawa's report [2]. In general, Helicobacter pylori infection and related severe atrophic gastritis and intestinal metaplasia are significantly associated with intestinal type non-cardia GC [9]. Lauren's diffuse type of GC, which is characterized by diffuse cellular infiltrating without gland formation, is more common in younger individuals [10]. While the detailed characteristics of SPC of the stomach was not disclosed in Maekawa et al. report, typical intestinal type GC might have predominated because of the high proportion of older individuals in the study. Due to the decreasing prevalence of Helicobacter pylori infection, the anatomical distribution of intestinal type GC has shifted from the distal to the proximal stomach around the gastro-esophageal junction [11]. The Cancer Genome Atlas project revealed four major genomic subtypes of GC and described the clinical characteristics of each subtype, including the location and the age at initial diagnosis [12]. While a minority of participants in the Cancer Genome Atlas project were from East Asian countries, distal GC was predominant type among East Asians, unlike that of the overall study cohort. Our practice including endoscopic monitoring for GC will be organized based on these subtypes in the near future. Anyway, the study by Maekawa and colleagues provides invaluable information for improving our clinical practice and provides a stimulus for research not only on age specific incidence of SPC after ER.

\section{References}

1. Yokoyama A, Kakiuchi N, Yoshizato T, et al. Age-related remodeling of oesophageal epithelia by mutated cancer drivers. Nature. 2019;565:312-7.

2. Maekawa A, Ishihara R, Iwatsubo T, et al. High incidence of head and neck cancers after endoscopic resection for esophageal cancer in younger patients. J Gastroenterol. 2019. https://doi.org/ 10.1007/s00535-019-01653-y.

3. Matsubara T, Yamada K, Nakagawa A, et al. Risk of second primary malignancy after esophagectomy for squamous cell carcinoma of the thoracic esophagus. J Clin Oncol. 2003; 1(21):4336-411.

4. Muto M, Nakane M, Katada C, et al. Squamous cell carcinoma in situ at oropharyngeal and hypopharyngeal mucosal sites. Cancer. 2004;15(101):1375-81.

5. Muto M, Minashi K, Yano T, et al. Early detection of superficial squamous cell carcinoma in the head and neck region and esophagus by narrow band imaging: a multicenter randomized controlled trial. J Clin Oncol. 2010;20(28):1566-72.

6. Morimoto H, Yano T, Yoda Y, et al. Clinical impact of surveillance for head and neck cancer in patients with esophageal squamous cell carcinoma. World J Gastroenterol. 2017;14(23):1051-8.

7. Katada C, Yokoyama T, Yano T, et al. Alcohol consumption and multiple dysplastic lesions increase risk of squamous cell carcinoma in the esophagus, head, and neck. Gastroenterology. 2016;151:860-9 (e7).

8. Abiko S, Shimizu Y, Miyamoto S, et al. Risk assessment of metachronous squamous cell carcinoma after endoscopic resection for esophageal carcinoma based on the genetic polymorphisms of alcoholdehydrogense-1B aldehyde dehydrogenase-2: temperance reduces the risk. J Gastroenterol. 2018;53:1120-30.

9. Uemura N, Okamoto S, Yamamoto S, et al. Helicobacter pylori infection and the development of gastric cancer. N Engl J Med. 2001;13(345):784-9.

10. Fenoglio-Preiser C, Carneiro F, Correa R, et al. Gastric carcinoma world health organization classification of tumors pathology and genetics of tumors of the digestive system. 2000; p. $39-52$

11. Hayakawa Y, Sethi N, Sepulveda AR, et al. Oesophageal adenocarcinoma and gastric cancer: should we mind the gap? Nat Rev. 2016;16:305-18.

12. Cancer Genome Atlas Research Network, et al. Comprehensive molecular characterization of gastric adenocarcinoma. Nature. 2014;513:202-9.

Publisher's Note Springer Nature remains neutral with regard to jurisdictional claims in published maps and institutional affiliations. 